\title{
Synergy between Sophorolipid Biosurfactant and SDS Increases the Efficiency of $P$. aeruginosa Biofilm Disruption
}

\author{
Bac V. G. Nguyen, Toshiki Nagakubo, Masanori Toyofuku, Nobuhiko Nomura,* and Andrew S. Utada*
}

Cite This: Langmuir 2020, 36, 6411-6420

Read Online

ABSTRACT: Biofilms are communities of bacteria encased in selfsecreted extracellular polymeric substances (EPS) that adhere stubbornly to submerged surfaces. Once established, these communities can cause serious chronic illnesses in medical settings, while they can promote corrosion and biofouling in industrial settings. Due to the difficulty of their removal, strongly oxidizing chemicals and detergents can be used to degrade and remove biofilms by killing the cells and degrading the matrix; however, the choice of compounds is limited in delicate environments due to the potential damage they may cause. In the case of detergents, most are synthesized from nonrenewable petrochemicals that have a degree of aquatic toxicity. There is a growing

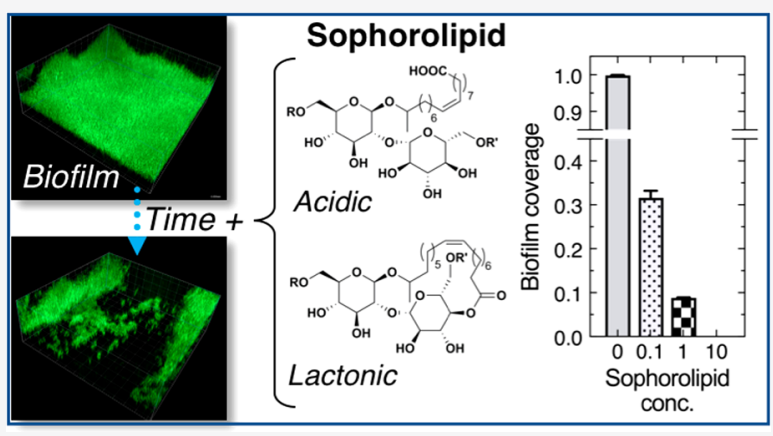
need to identify and characterize alternatives to synthetic surfactants.

Biosurfactants, which are surfactants produced by microorganisms, are a promising alternative since they can be synthesized from renewable resources, have low environmental toxicity, and have been shown to have higher degrees of specificity in the mechanism of action. Sophorolipids are a class of glycolipid surfactants produced by yeast that have demonstrated great promise due to large yields from renewable feedstocks and for antimicrobial properties; however, the effect of the application of sophorolipids to Gramnegative bacterial biofilms has not been well studied. We investigate the antibiofilm properties of sophorolipids by demonstrating its ability to cause the catastrophic disruption of Pseudomonas aeruginosa PAO1 biofilms in microfluidic channels. We show that while sophorolipids inflict little damage to the bacteria, they weaken the EPS biofilm matrix, leading to surface-detachment and breakup of the biofilm. Furthermore, we find that sophorolipids act cooperatively with the widely used surfactant, sodium dodecyl sulfate. When combined, concentrations $\sim 100$-fold lower than the minimum effective concentration, when used independently, recover potency. Biosurfactants are typically expensive to produce, thus our work demonstrates a means to improve efficacy while simultaneously reducing both cost and the amount of environmentally harmful substances used.

\section{INTRODUCTION}

Bacteria have two dominant lifestyles: as free-living, planktonic cells or as part of a dense aggregate, called a biofilm. Biofilms are found attached to submerged surfaces, encased in a selfsecreted matrix composed of a complex mixture of polysaccharides, extracellular DNA, and proteins; this is collectively termed extracellular polymeric substances (EPS). ${ }^{1,2}$ The EPS matrix covers and aids in adhering the colony to submerged surfaces while simultaneously providing protection to the encased bacteria from changing environmental conditions, chemical and physical attack, ${ }^{3}$ and predation. $^{4}$

The biofilm lifecycle is thought to be an adaptation to a variable environment, ${ }^{1,2}$ enabling bacteria to survive in a wide range of environments. ${ }^{1}$ Biofilms can form on nearly any wet surface from indwelling devices and implants, where they can cause chronic and recalcitrant infections, ${ }_{1,2,5,6}$ to the surfaces of potable water systems and reverse osmosis membranes, ${ }^{7,8}$ where they can foul, clog, and corrode the systems. ${ }^{9,10}$ Biofilms can be removed through a combination of mechanical and chemical attack; ${ }^{1,2}$ however, the removal strategy depends on a balance between the need for removal and damage to the underlying tissue or substrate. ${ }^{8}$ Dislodging them is challenging and costly. 5

Surfactants, which are widely used in cosmetics, hand soaps, and personal care products, ${ }^{11,12}$ are commonly used to suppress and disrupt biofilms. ${ }^{12,13}$ Due to their amphiphilicity, they can damage the integrity of the bacterial lipid membrane, leading to the loss of cell viability and the disruption of biofilms. ${ }^{8,14-16}$ Most common surfactants are synthesized from nonrenewable petrochemicals, have low biodegradability, and are toxic in the environment; ${ }^{17-19}$ these factors represent major drawbacks, thus strategies to improve their efficiency is highly desirable. Biosurfactants, which are surfactants synthe-

Received: March 6, 2020

Revised: May 12, 2020

Published: June 1, 2020 
sized by microorganisms, have steadily gained interest because they can be generated from sustainable feedstocks, are biodegradable, have lower environmental toxicity, and are effective at various $\mathrm{pH}$ 's and ionic strengths. ${ }^{20-24}$ In addition, they hold the promise of having novel properties with increased specificity due to their origin; microorganisms are known to secrete biosurfactants for use in signaling, ${ }^{21}$ as chemical repellents, ${ }^{25,26}$ to facilitate surface motility, ${ }^{27}$ and in biofilm dispersal. ${ }^{28}$

Sophorolipids are a biosurfactant secreted by different species of Candida during the fermentation of oils. They are among the most promising biosurfactants since they can be generated from renewable substrates in industrially viable yields. $^{20,22,24,29,30}$ Sophorolipids are a type of glycolipid $^{20,22}$ composed of a hydrophilic dimeric-sugar headgroup and a hydrophobic fatty acid tail that range in length from $\mathrm{C}_{16}$ to $\mathrm{C}_{24} \cdot{ }^{24,31}$ Importantly, the carboxyl group on the fatty acid can be either free or internally esterified, which results in two stable forms: acidic sophorolipids (AS) and lactonic sophorolipids (LS) (Figure 1).

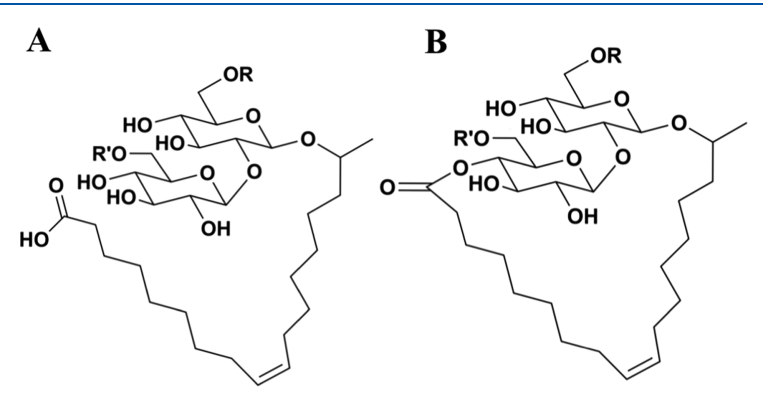

Figure 1. Schematic of the (A) acidic and (B) lactonic sophorolipid structures. $\mathrm{R}$ and $\mathrm{R}^{\prime}$ represent a $\mathrm{H}$ or an acetyl group.

The fermentation process generates complex mixtures of AS and LS. Furthermore, the AS and LS themselves are mixtures of similar molecules with different amounts of saturation and side groups. The specific makeup of the mixture depends on the species of Candida and the growth substrate used. Importantly, the physicochemical and biological properties of sophorolipids appears to depend strongly on the composition of the mixture.

Most reports have focused on the properties of the isolated components or on highly enriched mixtures containing $>95 \%$ in either AS or LS, respectively. The isolated components and highly enriched mixtures both demonstrate complex phase behavior in water; this contrasts with mixtures having ratios closer to 1 , which assemble only as micelles. ${ }^{32}$ In terms of biological effects, high-AS and high-LS content mixtures are both reported to have bactericidal effects on various Grampositive bacteria, ${ }^{33,34}$ where they are thought to increase the permeability of the cell membrane, leading to cell death. ${ }^{33,35,36}$

In contrast, mixtures where AS and LS are found in comparable ratios have demonstrated different and sometimes conflicting effects on microbial activity, which may depend on the ratio of AS to LS found in the mixture. ${ }^{23}$ Moreover, the number of studies that focus on the effects of sophorolipids on Gram-negative bacteria is limited. ${ }^{30,37}$ Due to the structural differences in the cell envelope between Gram-positive and Gram-negative, ${ }^{38,39}$ it is not evident how sophorolipids will interact with biofilm-forming Gram-negative bacteria. Isolated AS were shown to have some bactericidal effects on Escherichia $\operatorname{coli}^{33}$ and weak inhibitory effects on biofilm formation in Pseudomonas aeruginosa, both Gram-negative organisms. ${ }^{23}$ However, due to the limited number of studies, it is unclear how different ratios of AS/LS in sophorolipid mixtures will affect established biofilms. This highlights the need to characterize and clarify the antibiofilm properties of sophorolipids on biofilm-forming Gram-negative bacteria, which could have important implications for public health and biofouling in industrial settings.

Here, we demonstrate the antibiofilm properties of sophorolipids on established $P$. aeruginosa PAO1 biofilms grown in microfluidic channels. We target $P$. aeruginosa because of its importance as a human pathogen, a major cause of nosocomial infections, ${ }^{40}$ and as a model biofilmforming organism. ${ }^{41-45}$ We show that sophorolipids are better at disrupting PAO1 biofilms than is sodium dodecyl sulfate (SDS) and two polysorbate surfactants at similar concentrations, relative to the critical micelle concentration (CMC). Surprisingly, we find that sophorolipids have no antibacterial properties toward PAO1. To investigate the mechanism of action, we utilize a PAO1 mutant that overexpresses the EPS matrix, finding that sophorolipids appear to simultaneously weaken the adhesion between the biofilm and underlying glass surface as well as the internal cohesiveness of the biofilm. Finally, we describe a synergism between sophorolipids and SDS: used in combination, the mixture is able to disrupt PAO1 biofilms more effectively than the individual components and at significantly lower concentrations than the lowest effective concentration of either surfactant, when used individually.

\section{EXPERIMENTAL SECTION}

Materials. Commercially available sophorolipids (CAS no. 1573124-58-9), sold as ACS-Sophor, were purchased from Allied Carbon Solutions (Tokyo, Japan) at a concentration of 51.2 wt \% $(512 \mathrm{mg} / \mathrm{mL})$ dissolved in water. Sophorolipids were secreted during the fermentation of Madhuca oil by Candida bombicola and contain mixtures of AS and LS, which were used without further purification. The AS/LS ratio is listed as 3.8:6.2. Since the ACS-Sophor sophorolipids are composed of a mixture of different compounds, we refer to them collectively as SLx. We purchased sodium dodecyl sulfate (CAS no. 151-21-3), polyoxyethylene (20) sorbitan monolaurate (Tween 20) (CAS no. 9005-64-5), polyoxyethylene (80) sorbitan monolaurate (Tween 80) (CAS no. 9005-65-6), formate (CAS no. 64-18-6), 2-propanol (CAS no. 67-63-0), and sodium chloride from Nacalai Tesque (Kyoto, Japan). Fluorescein isothiocyanate (FITC)-conjugated Hippeastrum hybrid lectin (amaryllis) (HHA) was purchased from EY Laboratories, Inc. (San Mateo, CA, USA). DAPI was purchased from Thermo Fisher Scientific (Waltham, MA, USA). BactoAgar, BactoTryptone, and BactoYeast Extract were purchased from Becton-Dickinson (Baltimore, MD, USA). Polydimethylsiloxane (PDMS) was purchased as a Sylgard 184 silicone elastomer kit from Dow Corning Toray (Tokyo, Japan). KMPR photoresist was purchased from MicroChem (Westborough, MA, USA).

Liquid Chromatography-Tandem Mass Spectrometry (LCMS/MS). LC-MS/MS analysis was carried out using a Nexera X2 system and an LCMS-8050 (Shimadzu, Kyoto, Japan) equipped with a TSK-gel ODS-120H $3 \mu \mathrm{m} 2.0 \times 150 \mathrm{~mm}$ (Tosoh Co., Ltd., Tokyo, Japan). The sophorolipids were analyzed under the following conditions: flow rate $=0.2 \mathrm{~mL} / \mathrm{min}$; temperature $=40{ }^{\circ} \mathrm{C}$; solvent A, $0.1 \%(\mathrm{v} / \mathrm{v})$ formate in $\mathrm{H}_{2} \mathrm{O}$; and solvent $\mathrm{B}, 0.1 \%(\mathrm{v} / \mathrm{v})$ formate in 2-propanol. After column equilibration with $100 \%$ solvent $\mathrm{A}, 2 \mu \mathrm{L}$ of sample was injected into the column. The sample was eluted from the column with the following gradient: $0-20 \mathrm{~min}, 0-100 \%$ solvent $\mathrm{B}$; $20-25 \mathrm{~min}, 100 \%$ solvent $\mathrm{B} ; 25-30 \mathrm{~min}, 0 \%$ solvent $\mathrm{B}$. The precursor 
ions and the fragment ions were detected in positive ion mode. Previous reports ${ }^{31}$ were used to identify the sophorolipid structures.

Surface Tensiometry and Micelle Size Distribution. The surface tension of the surfactant solutions was measured using the Wilhelmy plate method. Surfactants were dissolved in $100 \mathrm{~mL}$ of deionized (DI) water and then measured as a function of the mass percentage using the FACE automatic surface tensiometer (Kyowa Interface Science Co., Ltd., Tokyo Japan) at $25.2{ }^{\circ} \mathrm{C}$. Each measurement was performed in triplicate on the same day and then repeated using a different sample on different days.

Using dynamic light scattering (DLS), we measured the micelle size distribution of the surfactants with a Zetasizer Nano ZS (Malvern Panalytical Ltd., U.K.) at $25.2{ }^{\circ} \mathrm{C}$. All surfactants were dissolved in Milli-Q ultrapure water at a concentration of $1 \mathrm{wt} \%$ and then filtered through a syringe filter with a Millipore membrane $(0.45 \mu \mathrm{m}$ pore diameter) prior to measurement. A concentration of $1 \mathrm{wt} \%$ is well above the CMCs of all surfactants used. The wt \% concentrations were normalized by the CMCs for the respective surfactants and are listed in Table S2.

Bacterial Strains. We used the Pseudomonas aeruginosa PAO1 wild-type (WT) strain ${ }^{46}$ and the $\Delta w s p F$ deletion mutant in the PAO1 background. The $\Delta w s p F$ deletion mutant was constructed using homologous recombination. Approximately $800 \mathrm{bp}$ fragments upstream and downstream of $w s p F$ were amplified by PCR using $\Delta$ wspFF1 (5'-GCTCTAGAAGATCGGCGAGGAGGCCTAC$\left.3^{\prime}\right) / \Delta$ wspFR1 (5'-GGCTAATCGCTCAACCCTGTGCTTCTCCGA-3') and $\Delta$ wspFF2 (5'-AGGGTTGAGCGATTAGCCGGGTTCGACTC-3')/ ${ }^{\prime}$ wspFR2 ( $5^{\prime}$-CGGAATTCTCCGAGGAGCGACTGCAGCC-3) primer sets. The two DNA fragments were assembled using overlap extension PCR. The resulting product was digested with $\mathrm{XbaI}$ and EcoRI and cloned into pG19II to generate pG19wspF. $\mathrm{pG} 19 \mathrm{wspF}$ was used for homologous recombination to delete $w s p F$, and the deletion was confirmed by PCR. Deletion of the $w s p F$ gene leads to the constitutive activation of $W s p R$, which results in the intracellular accumulation of cyclic diguanylate (c-di-GMP). The wsp operon functions as a signal transduction pathway that regulates biofilm formation through the modulation of c-di-GMP, which has been shown to be a biofilm master regulator. The $\triangle w s p F$ strain produces significantly thicker biofilms with respect to the WT due to the overexpression of polysaccharide components in the EPS. ${ }^{47}$

Bacterial Culture Conditions and Growth Assays. Lysogeny broth (LB) was prepared by mixing BD BactoTryptone (final concentration $10 \mathrm{~g} / \mathrm{L}$ ), BD BactoYeast Extract (final concentration 5 $\mathrm{g} / \mathrm{L}$ ), and $\mathrm{NaCl}$ (final concentration $5 \mathrm{~g} / \mathrm{L}$ ) in deionized (DI) water and was subsequently placed in an autoclave. LB agar was made by adding BD BactoAgar (final concentration $15 \mathrm{~g} / \mathrm{L}$ ) to LB followed by being treated in an autoclave.

The bacteria were grown by streaking frozen stocks, stored at -80 ${ }^{\circ} \mathrm{C}$, on LB agar plates, followed by overnight $(12-16 \mathrm{~h})$ incubation at $37^{\circ} \mathrm{C}$. One or two colonies were subsequently picked up and used to inoculate a test tube containing $4 \mathrm{~mL}$ of $\mathrm{LB}$, which was then incubated overnight at $37^{\circ} \mathrm{C}$ in an orbital shaker at $190 \mathrm{rpm}$; this is enough time for the culture to reach the stationary phase. We then made a subculture by transferring $100 \mu \mathrm{L}$ of this stationary-phase culture to $4 \mathrm{~mL}$ of fresh $\mathrm{LB}$ and then incubating for $\sim 4-6 \mathrm{~h}$ at $37^{\circ} \mathrm{C}$ in an orbital shaker. We monitor cell growth by measuring the absorbance of the cell culture at $600 \mathrm{~nm}\left(\mathrm{OD}_{600}\right)$ using a NanoDrop 2000c spectrophotometer (Thermo Scientific, Waltham, MA, USA). The initial $\mathrm{OD}_{600}$ of the subculture was $\sim 0.01$. After approximately 4-6 $\mathrm{h}$ of incubation, the subculture reaches the middle of the exponential growth phase. We used this culture method unless otherwise noted.

Microfluidic Device Fabrication and Biofilm Growth in Microchannels. We fabricated microfluidic devices in PDMS using standard soft lithography techniques. ${ }^{48}$ The devices were designed with four parallel growth channels and a single inoculation port leading to each channel, individually. Prior to use, we autoclaved, degassed, and filled the microfluidic device with sterile $10 \% \mathrm{LB}$. We diluted the midexponential phase culture to an $\mathrm{OD}_{600}$ of 0.01 in the same medium and used $\sim 400 \mu \mathrm{L}$ to inoculate the device through inlet (i) using a $1 \mathrm{~mL}$ syringe connected to a sterile silicone tube. The newly introduced cells were imaged to ensure sufficient cell coverage and subsequently incubated for $30 \mathrm{~min}$ under static conditions to allow the bacteria to attach to the surface of the device. We then infused 10\% LB through the inlets (ii-v), which supplies the growth channels, at a flow rate $(Q)$ of $100 \mu \mathrm{L} / \mathrm{h}$ using a positive displacement syringe pump (Harvard Apparatus, Holliston, MA, USA). The microfluidic devices were incubated at $30{ }^{\circ} \mathrm{C}$ using a microscope stage heater (Tokai Hit, Shizuoka, Japan). Images of biofilm development were acquired continuously in all four channels every minute for $12-16 \mathrm{~h}$ on an inverted microscope (Carl Zeiss Axio Observer Z1).

After generating the biofilms in the channels, using a three-way valve, we then began the infusion of fresh medium containing the surfactants into the channels. We recorded time-lapse images in each channel, every minute, for $5 \mathrm{~h}$ on an inverted microscope.

Biofilm Surface Coverage Calculation. We processed the bright-field image sequences using ImageJ to segment and count cells and/or cell clusters. We applied a threshold to the image sequences, extracted the number of bacterial clusters on the surface, and then, using "Analyze Particle", calculated the percentage of the surface area occupied by bacteria in each image, $A_{\text {covered }}$, as a function of time, $t$. We normalized $A_{\text {covered }}$ by the total area of the field of view, $A_{0}$. Each point in the coverage plots is the average $A_{\text {covered }}$ calculated from 10 separate images to ensure that the bacteria were actually attached to the surface and not floating through the field of view. These images were taken $1 \mathrm{~min}$ apart and were centered at $\pm 5 \mathrm{~min}$ around each hour. These tests were done in triplicate with different starting cultures.

Bacteriostatic, Bactericidal, and Colony-Forming-Unit (CFU) Viability Assays. We measured the bacteriostatic properties of the surfactants by measuring the growth of PAO1 in the presence of surfactants. We added different concentrations of surfactants to the subculture step described earlier and then measured the absorbance as a function of time. The test tubes were incubated at $37{ }^{\circ} \mathrm{C}$ in an orbital shaker at $190 \mathrm{rpm}$.

To evaluate the bactericidal properties, we allowed subcultures to reach $\mathrm{OD}_{600} \approx 0.15$ before adding the surfactants. We measured the $\mathrm{OD}_{600}$ as a function of time as the test tubes were incubated at $37^{\circ} \mathrm{C}$ in an orbital shaker at $190 \mathrm{rpm}^{49}$

We measured the number of viable cells by counting the number of colony-forming units (CFU) found in the liquid culture. Here, we added SLx directly to the subculture and incubated for $12 \mathrm{~h}$. We then serially diluted the cultures in fresh LB and immediately plated the cells by uniformly spreading $100 \mu \mathrm{L}$ of the suspension onto LB agar plates. We incubated the plates overnight and then counted the number of colonies on plates with well-separated colonies, giving the $\mathrm{CFU} / \mathrm{mL}$.

$\Delta w s p F$ Biofilm Formation and $\mathrm{OD}_{600}$ Measurement. We cultured the $\Delta w s p F$ strain in the same manner as described earlier for WT; however, we incubated the subculture for $12 \mathrm{~h}$ in a linear-shaker incubator $\left(190 \mathrm{rpm}, 37^{\circ} \mathrm{C}\right)$ to generate biofilms attached to the test tubes. After biofilm development, we added different concentrations of SLx to the test tubes and incubated for an additional $5 \mathrm{~h}$. To measure the number of planktonic cells in the test tubes, we halted agitation and waited $5 \mathrm{~min}$ before sampling $500 \mu \mathrm{L}$ of the culture to measure the respective $\mathrm{OD}_{600}$.

Lectin Staining and Confocal Imaging. To label the polysaccharides in the $\Delta w s p F$ biofilms, we scraped biofilm from the air-liquid interface using a pipet tip. The sticky biofilm sample was suspended in $1 \mathrm{~mL}$ of phosphate-buffered saline (PBS) containing $100 \mu \mathrm{g} / \mathrm{mL}$ FITC-HHA lectin and $300 \mathrm{nM}$ DAPI and was subsequently incubated for $60 \mathrm{~min}$ at room temperature. The sample was then placed between pieces of cover glass and imaged using a confocal microscope. We used Imaris software (AG, Zurich, Switzerland) to threshold and calculate the fluorescently labeled volume from $40 z$ slices ( $80 \mu \mathrm{m}$ range) of the biofilm. We normalized the FITC-lectin-labeled volume by the volume of cells labeled with DAPI. 


\section{RESULTS AND DISCUSSION}

LC-MS/MS Sophorolipid Characterization. We characterize the molecular structure of the SLx used in this study since its reported effect on Gram-positive and -negative bacteria can depend on the specific composition. ${ }^{23}$ Using LC-MS/MS, the analysis of the sophorolipid chromatogram reveals the presence of different components with various side groups on the sugar moieties as well as fatty acid tails, which differ in length and in the degree of saturation (Figure S1 and Table S1). The relative intensity of the AS peaks compared to that of the LS peaks confirms that the ratio of AS to LS reported in the mixture is consistent with our results.

Surfactant Surface Properties. We compare the effects of SLx with SDS, Tween 20, and Tween 80, which are common surfactants that are frequently used as additives in foods, cosmetics, personal care products, and pharmaceuticals. ${ }^{15,17,50}$ SDS is an anionic surfactant that damages bacterial membranes, whereas the tween surfactants are nonionic surfactants that are reported to have antibiofilm properties. ${ }^{15,51}$

We first determine the respective CMCs of the surfactants by measuring the surface tension $(\gamma)$ since it can determine bioavailability ${ }^{52,53}$ and affect antibacterial properties ${ }^{52}$ and is correlated with aquatic toxicity (Figure 2). ${ }^{54}$ In addition to the

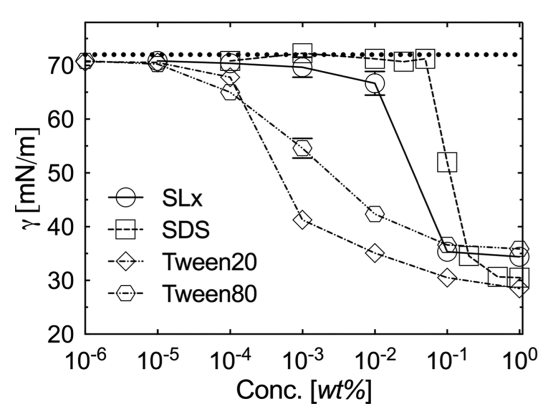

Figure 2. Surface tension $(\gamma)$ of $(O)$ SLx, $(\square)$ SDS, $(\diamond)$ Tween 20, and $(\square)$ Tween 80 dissolved in deionized (DI) water, measured as a function of concentration. The error bars represent one standard deviation (SD) from three measurements. Error bars smaller than the symbol are omitted for clarity. The horizontal dashed line shows the surface tension of DI water $(72 \mathrm{mN} / \mathrm{m})$. Each measurement is the average value of three measurements.

CMC, we use DLS to measure the micelle size distributions, shown in Figure S3. The CMCs we measure for SDS, ${ }^{55}$ Tween $20,{ }^{56}$ and Tween $80^{56}$ are in agreement with previous studies, whereas the CMC for SLx is $10^{-1}$ wt \%, which is $\sim 10$ times larger than previously reported (Table 1). ${ }^{57}$ This difference could arise from differences in the strain of Candida used to synthesize the sophorolipids as well as the growth substrate. $^{58,59}$

Effect of Surfactants on Biofilms Grown in Microchannels. To test the effect of the surfactants on established biofilms, we use $P$. aeruginosa PAO1, which secretes various adhesive molecules that enable its biofilms to stubbornly adhere to surfaces, ${ }^{60}$ where it can accumulate and disrupt flow through channels. ${ }^{10,61}$ We use microfluidics to test the surfactants since it provides a convenient platform for mimicking pores and gaps found in industrial filters, reverse osmosis membranes, ${ }^{7,62}$ catheters, and stents ${ }^{33}$ while enabling the simple optical readout of the state of the biofilm. We design our device with four separate, parallel, microfluidic channels and a single port for simultaneous inoculation (Figure
Table 1. Critical Micelle Concentration (CMC) of the Surfactants Used in These Tests Given in wt \% and Equivalent Molar Concentration ${ }^{a}$

\begin{tabular}{lll} 
& \multicolumn{2}{c}{ CMC } \\
\cline { 2 - 3 } surfactant & wt \% & $\mathrm{mM}$ \\
SLx & 0.1 & 1.5 \\
SDS & 0.2 & 6.9 \\
Tween 20 & 0.001 & 0.008 \\
Tween 80 & 0.002 & 0.02
\end{tabular}

${ }^{a}$ We estimate the average MW of SLx by taking a weighted average of the MWs of each component in the mixture.

3A,B). By inoculating all channels simultaneously, we ensure that similar numbers of biofilm-founding bacteria attach to the surface of each channel, thus synchronizing growth therein and simplifying analysis.

To generate biofilms, we supply the attached bacteria with growth medium at a constant flow rate $(Q)$ and monitor biofilm development by recording time-lapse images. From these images, we extract the area covered by bacteria $\left(A_{\text {covered }}\right)$ as a function of time (Figure 3C). A three-dimensional confocal image of a typical PAO1 biofilm grown overnight in the channels is shown in Figure 3D. Upon biofilm establishment, $A_{\text {covered }} / A_{0}=1$. At this point, we begin the infusion of medium containing surfactants, at a concentration of $C$, while continuing to image the biofilm. We find that although all four surfactants demonstrate varying degrees of efficacy in PAO1 biofilm disruption, at all concentrations SLx demonstrates the strongest effects. Micrographs showing the state of the biofilm before and after the introduction of $0.1 \mathrm{wt} \%$ SLx are shown in Figure 4A. For each tested SLx concentration, we find that $A_{\text {covered }}$ decreases continuously throughout the course of the experiment. This contrasts with SDS, which causes an initial decrease in $A_{\text {covered }}$ followed by a plateau in the rate of disruption. Tween 20 demonstrates only weak disruptive effects at all tested concentrations, whereas Tween 80 begins to cause significant disruption only at 1 wt $\%$. The change in $A_{\text {covered }}$ for different wt $\%$ concentrations is plotted as a function of time in Figure 4B. Pre- and post-treatment micrographs are shown at two concentrations for each surfactant in Figure S3.

Since biofilm disruption may strongly depend on the aggregative properties of the surfactants, ${ }^{52,53}$ we normalize the concentration of each surfactant, $C$, by its respective CMC (Table 1) and plot the bacterial coverage at the end of the microchannel experiment $(t=5 \mathrm{~h})$ as a function of the normalized surfactant concentration $\left(C^{\prime}=\frac{C}{C M C}\right)$. This normalization allows us to compare the relative effects based on CMC equivalents. We find that SLx disrupts $\sim 70 \%$ of the biofilm at $C_{\text {SLx }}^{\prime}=10^{-1}$ and nearly $90 \%$ at $C_{\text {SLx }}^{\prime}=1$. In contrast, both Tween 20 and 80 are ineffective at these normalized concentrations, while SDS demonstrates intermediate effects (Figure 4C). Thus, although large surfactant concentrations relative to the CMC may begin to disrupt PAO1 biofilms, SLx demonstrates a stronger antibiofilm effect at significantly lower values than for the other chemical surfactants.

Bacteriostatic and Bactericidal Properties of Surfactants. To clarify the role of SLx in biofilm disruption, we evaluate the bacteriostatic and bactericidal effects of the surfactants, which may play a role in biofilm disruption. We track cell growth by measuring absorbance $\left(\mathrm{OD}_{600}\right)$ in liquid 

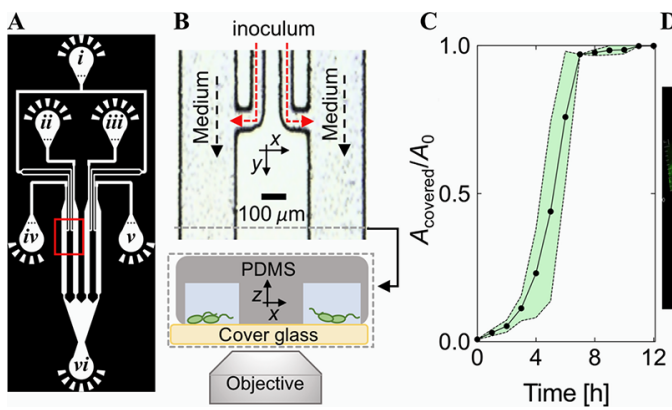

D

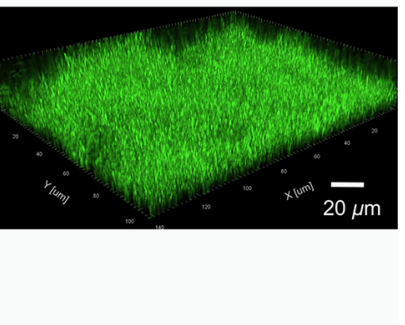

Figure 3. Biofilms are grown in microfluidic channels under continuous flow. (A) Schematic of the microfluidic device used. Inlet (i) is used to inoculate each channel with the same bacterial culture, while the four inlets (ii-v) are used to infuse different solutions. The outlet is labeled (vi). (B) (Upper) A micrograph of the red box in (A) shows two growth channels and their inoculation ports (smaller channels). The growth-channel dimensions are $200 \mu \mathrm{m}$ (width) $\times 80 \mu \mathrm{m}$ (height) $\times 10^{4} \mu \mathrm{m}$ (length). (Lower) Cross-sectional schematic of the channels at the dashed line. (C) Surface area covered by bacteria $\left(A_{\text {covered }}\right)$, normalized by the total area $\left(A_{0}\right)$ of the field of view, measured as a function of time. The line and dots represent the average of three experiments, while the filled region represents one SD from three experiments. (D) Confocal image of a typical mature biofilm taken at $12 \mathrm{~h}$. The bacteria are labeled with the DNA stain DAPI but are shown here in green for clarity. In each of these tests, the medium is $10 \% \mathrm{LB}$, the flow rate is $100 \mu \mathrm{L} / \mathrm{h}$, and the entire device is incubated at $30^{\circ} \mathrm{C}$.
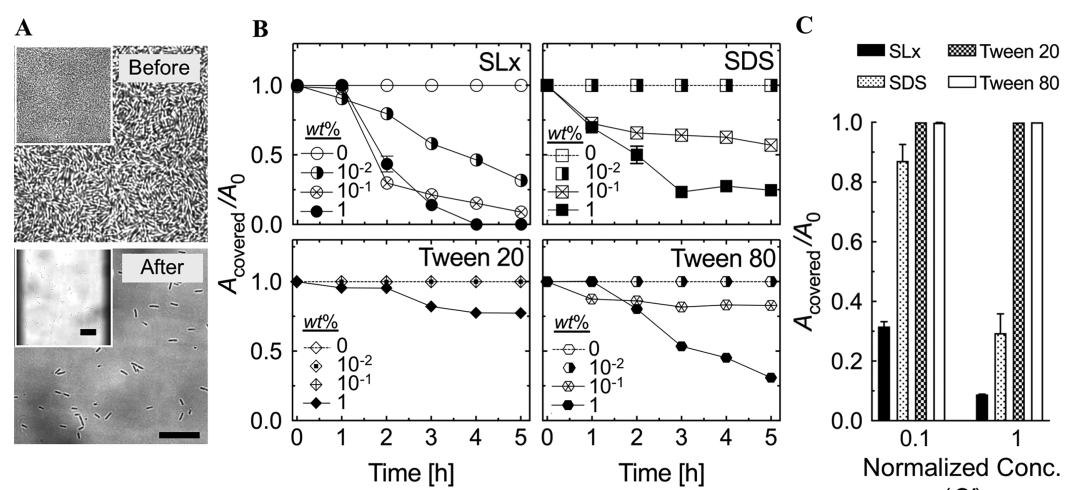

$\left(C^{\prime}\right)$

Figure 4. Change in surface coverage of mature PAO1 biofilms grown in microfluidic channels measured as a function of time for different surfactant concentrations. (A) Image of channels pre- and post-treatment with $0.1 \mathrm{wt} \%$ SLx for $5 \mathrm{~h}$ with dimensions of $89 \times 89 \mu \mathrm{m}^{2}$. (Insets) The whole channel is shown, which has dimensions of $133 \times 133 \mu \mathrm{m}^{2}$. The bars are $10 \mu \mathrm{m}$. (B) Surface area covered by bacteria $\left(A_{\text {covered }}\right)$, normalized by the total area $\left(A_{0}\right)$ of the field of view, measured as a function of surfactant-treatment time. The symbols represent different surfactant concentrations. Each experiment was done in triplicate, and the error bars represent one SD. Error bars smaller than the symbol are omitted. (C) Normalized bacterial surface coverage at $5 \mathrm{~h}$ shown for two concentrations normalized by the respective CMCs given in Table 1 . The values for SDS are estimated by interpolating the $A_{\text {covered }}\left(\right.$ from (B)) as a function of SDS concentration using an exponential decay $\left(R^{2}=0.99\right)$. The carrier medium is $10 \% \mathrm{LB}$, and the channels are incubated at $30{ }^{\circ} \mathrm{C}$. The flow rate is $100 \mu \mathrm{L} / \mathrm{h}$.

culture containing different surfactant concentrations in LB. We find that SLx, Tween 20, and Tween 80 have no inhibitory effects on PAO1 growth. In contrast, SDS inhibits growth in a concentration-dependent manner: PAO1 growth is significantly inhibited for $C_{S D S}^{\prime} \geq 5 \times 10^{-1}$, although there is a gradual upward trend in the $\mathrm{OD}_{600}$ after $\sim 10 \mathrm{~h}$ (Figure $5 \mathrm{~A}$ ). To measure bactericidal effects, we test a concentration of $1 \mathrm{wt}$ $\%$, which is well above the CMC for each surfactant. The $1 \mathrm{wt}$ $\%$ concentration corresponds to $C_{\mathrm{SLx}}^{\prime}=\sim 10, C_{\mathrm{SDC}}^{\prime}=\sim 5$, $C_{\text {Tween20 }}^{\prime}=10^{3}$, and $C_{\text {Tween80 }}^{\prime}=\sim 5 \times 10^{2}$. We measure changes in absorbance upon addition of the surfactants to liquid culture in the midexponential phase; here, decreases in $\mathrm{OD}_{600}$ would correspond to surfactant-mediated cell lysis. We find that SLx, Tween 20, and Tween 80 have no bactericidal effects, whereas SDS initially causes a reduction in the $\mathrm{OD}_{600}$ over the first $3 \mathrm{~h}$. We note that $5 \mathrm{~h}$ after addition, the $\mathrm{OD}_{600}$ begins to increase (Figure S4). The initial decrease in $\mathrm{OD}_{600}$ indicates cell lysis immediately after the addition of SDS, while the increase in $\mathrm{OD}_{600}$ after $\sim 5 \mathrm{~h}$ indicates recovery and regrowth.

The absorbance is useful for monitoring the number of cells in solution, but it provides no information on cell viability.
Since SLx demonstrates the strongest effects on disrupting PAO1 biofilms, we measure the number of PAO1 colonyforming units (CFU) after $12 \mathrm{~h}$ of liquid culture in SLx-laden LB medium to determine if SLx has deleterious effects on the number of viable cells. Confirming the absorbance data, the $\mathrm{CFU} / \mathrm{mL}$ shows that SLx has no adverse effects but instead appears to slightly increase the $\mathrm{CFU} / \mathrm{mL}$ as a function of concentration (Figure 5B).

These results suggest that the biofilm disruption demonstrated by Tween 20 and 80 is likely unrelated to their bacteriostatic and bactericidal effects. In fact, their presence appears to promote PAO1 growth (Figure 5). In contrast, SDS, which is known to cause cell lysis, transiently inhibits growth and exhibits bactericidal properties in a concentrationdependent manner. The regrowth of PAO1 in the presence of SDS is surprising but may be due to the accumulation of SDS-degrading enzymes, which have been reported in other species of $P$. aeruginosa. ${ }^{63}$ These two competing effects may be the cause of the decrease, followed by the plateau in $A_{\text {covered }}$ observed at all SDS concentrations (Figure 4B). SLx, like Tween 20 and 80, exhibits no inhibitory effects on PAO1 

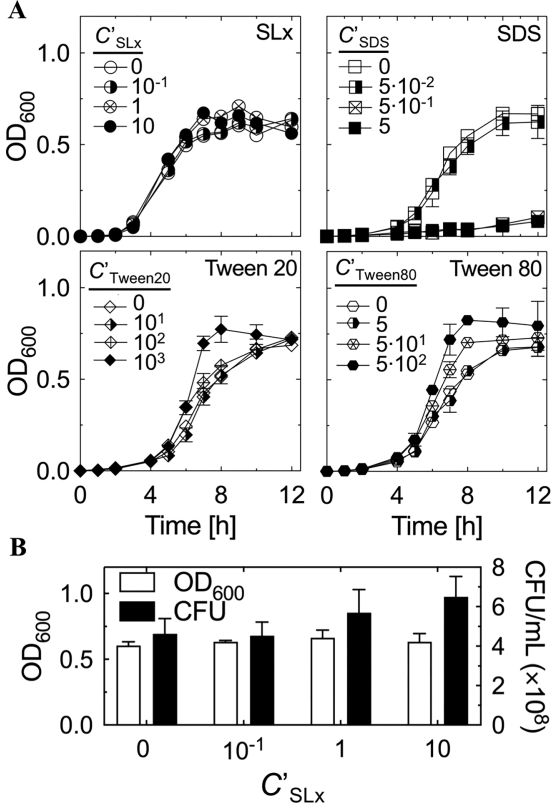

Figure 5. (A) PAO1 growth curves in LB at different surfactant concentrations, measured using absorbance $\left(\mathrm{OD}_{600}\right)$. The listed concentrations are normalized by the respective CMC. (B) Comparison of the $\mathrm{OD}_{600}$ and $\mathrm{CFU}$ at $12 \mathrm{~h}$ in LB with different SLx concentrations. Each experiment was done in triplicate, and the error bars represent one SD. Error bars smaller than the symbols are omitted for clarity.

growth, although it has the strongest antibiofilm properties. Moreover, despite the fact that sophorolipids have been shown to have bactericidal effects on both Gram-positive (S. aureus) and Gram-negative (E. coli) bacteria, ${ }^{33,35}$ the differences we observe are likely due to differences in potency that depend on the specific bacterium used and the composition of the SLx mixture. ${ }^{57}$ Importantly, these results suggests that the mechanism of SLx-mediated biofilm disruption is independent of its growth-inhibitory or bactericidal properties.

Mechanism of Biofilm Disruption. Alternatively, since the polysaccharides in the EPS matrix of PAO1 biofilms are adhesive and provide structural support for the biofilm community, we hypothesize that there is an interaction between the SLx and EPS polysaccharides. To test this interaction, we utilize the deletion strain PAO1 $\Delta w s p F$. This mutant overexpresses two structurally important polysaccharides, psl and pel, ${ }^{47}$ due to increased intercellular levels of the biofilm master regulator, c-di-GMP. Under typical shakingculture growth conditions, unlike the WT, $\Delta w s p F$ mutants form both thick biofilms that adhere to the glass test tubes around the air-liquid interface and biofilm aggregates in the culture medium that sediment to the bottom of the tube $\left(C_{\mathrm{SLx}}^{\prime}\right.$ $=0$ in Figure 6A,D). When we add SLx to the liquid culture and continue shaking incubation, we see a dose-dependent disruption of the biofilm; after $5 \mathrm{~h}$ of co-incubation, the amount of biofilm attached to the interface is inversely proportional to the SLx concentration (Figure 6A). WT grown in the same manner does not generate biofilms attached near the air-liquid interface nor does it aggregate into large clusters that settle in the test tubes (Figure S5).

The thinning of the biofilm at the air-liquid interface suggests that the addition of SLx weakens the EPS matrix, which in PAO1 $\Delta w s p F$ and WT largely consists of polysaccharides. ${ }^{47}$ To examine the change in the EPS, we collect biofilms from the air-liquid interface and label the polysaccharides in the EPS using an FITC conjugated lectin. ${ }^{64}$ We integrate the intensity from the confocal images to calculate the fluorescently labeled volume. We normalize by the DAPI-labeled cells, corresponding to the amount of polysaccharide per cell present in the biofilm (Figure 6B). We find that the untreated sample has a larger volume of labeled polysaccharide than the biofilm treated with a concentration of $C_{\text {SLx }}^{\prime}=10$ (Figure 6C).

In addition to the biofilms formed at the air-liquid interface, the sedimented biofilm clusters at the bottom of the tubes offer another opportunity to observe the effects of SLx on $\Delta w s p F$ biofilms. After treatment, we transfer the contents of the test tubes into six-well plates for imaging; here, we find a decrease in the size and density of the biofilm aggregates (Figure 6D). Visual inspection of the sedimented aggregates indicates that with increasing SLx concentration, the optical density of the culture may decrease due to aggregate breakup (Figure 6D). However, when we measure the absorbance of these cultures (Figure 6A), carefully avoiding the aggregates as we sample the liquid, we find large increases in the $\mathrm{OD}_{600}$ that vary as a function of the SLx concentration, as shown in Figure 6E (also Figure $6 \mathrm{~A}$ ). This increase in $\mathrm{OD}_{600}$ indicates a large increase in the number of planktonic cells.
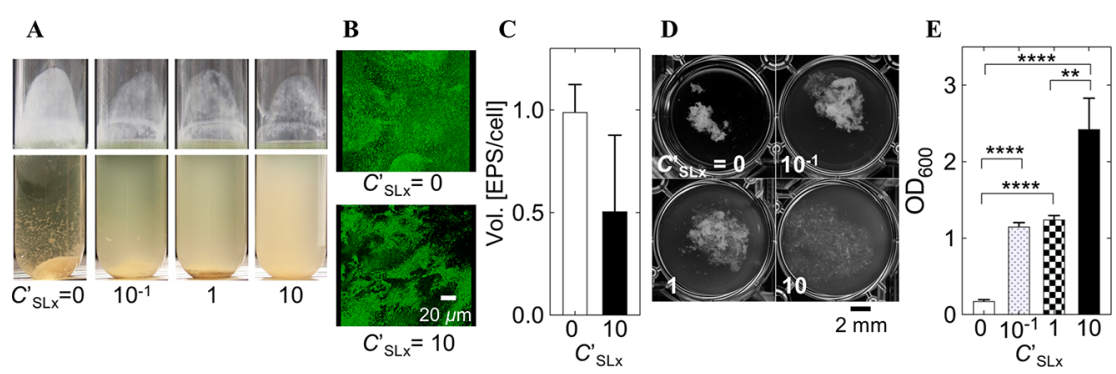

Figure 6. Effect of SLx on $\Delta w s p F$ biofilms in liquid culture. (A) Images of $\Delta w s p F$ biofilms cultured overnight in glass test tubes, subsequently treated with different concentrations of SLx for $5 \mathrm{~h}$ under continued shaking incubation. The viewing angle is held constant for the upper and lower sections of the test tubes. (B) Post-treatment biofilms collected from the air-liquid interface labeled with an FITC-HHA lectin and imaged using a confocal microscope. The biofilms are taken from the tubes with concentrations of 0 and 10 [wt \%/CMC] SLx. A single plane is shown. (C) Volume of the lectin-labeled biofilm calculated from the fluorescence intensity in (B). The error bars represent one SD from three experiments. (D) The contents of the test tubes in (A) are transferred to six-well plates and imaged. (E) Post-treatment $\mathrm{OD}_{600}$ of the liquid cultures, indicating planktonic cells, in different concentrations of SLx. We take care to avoid the aggregates. The statistical significance is calculated using the unpaired $t$-test $(n=3, * * P<0.01, * * * P<0.001, * * * * P<0.0001)$. 
Together, these results suggest that SLx weakens the adhesiveness of the $\Delta w s p F$ biofilm matrix to glass surfaces as well as the cohesiveness within the biofilms. This weakening leads to a detachment and thinning of the biofilm at the airliquid boundary, which is evident from the images of the test tubes. At the same time, as the EPS matrix weakens and breaks apart, large numbers of cells are freed from the biofilm, causing the absorbance to increase. The disruptive effects demonstrated on $\Delta w s p F$ biofilms are comparatively more destructive on WT, which generate less EPS: when $C_{\mathrm{SLx}}^{\prime}=1$ or $C_{\mathrm{SLx}}^{\prime}=10$ is applied to $\Delta w s p F$, residual biofilm remains attached at the airliquid interface (Figure 6A), whereas these concentrations disrupt $\sim 90$ and $100 \%$ of the WT biofilm in the microchannels, respectively $(\otimes$ and $\bigcirc$, Figure 4B). These differences are likely the result of a lower secretion of polysaccharide and, consequently, weaker biofilms in WT compared to that of the $\Delta w s p F$ mutant. $^{47}$

SLx and SDS Synergism. We attempt to improve the efficiency of SLx-mediated biofilm disruption by reducing the concentration necessary to achieve the antibiofilm activity demonstrated in Figure 4B. We do so by combining SDS with SLx since SDS demonstrates the strongest disruptive effects at the lowest CMC-equivalent concentrations of the synthetic surfactants that we tested. We choose a few sets of concentrations: one pair that has moderate activity, $C_{\mathrm{SLx}}^{\prime}=$ $10^{-1}$ and $C_{S D S}^{\prime}=5 \times 10^{-1}$, another pair at 10 -fold-lower concentrations, and the permutations of these concentrations. We characterize these mixtures first by measuring $\gamma$, finding that surface tension decreases significantly despite the fact that the concentrations of both surfactants are lower than their respective CMCs (Table 2). This effect may be similar to the

\section{Table 2. Surface Tension of SLx and SDS Mixtures ${ }^{a}$}

$\begin{array}{lcccc}{\left[\mathrm{C}_{\text {SDS }}^{\prime}\right](\downarrow)} & {\left[\mathrm{C}_{\text {SLx }}^{\prime}\right](\rightarrow)} & 0 & 10^{-2} & 10^{-1} \\ 0 & & 71.3 \pm 0.5 & 71.2 \pm 0.9 & 68.5 \pm 1.1 \\ 5 \times 10^{-2} & & 72.7 \pm 0.3 & 47.1 \pm 0.8 & 36.3 \pm 0.5 \\ 5 \times 10^{-1} & & 52.0 \pm 1.0 & 40.5 \pm 1.4 & 36.1 \pm 0.6\end{array}$

${ }^{a}$ Each value was measured from a separately prepared solution, measured in triplicate. The surface tension values are given in units of $\mathrm{mN} / \mathrm{m}$.

synergistic depression of $\gamma$ reported for the combination of synthetic surfactants with alkylglycosidic surfactants, which share some structural similarities with sophorolipids. ${ }^{65}$

The decrease in $\gamma$ suggests a possible benefit to mixing, which we use as a guide as we return to our microchannel experiments to evaluate the biofilm disruption properties. However, we note that surface tension alone is not a good predictor of biofilm removal activity: both Tween 20 and 80 demonstrate no ability to remove biofilms at a $1 \mathrm{CMC}$ equivalent (Figure 4C) and require much higher concentrations to begin to disrupt biofilms. In two separate tests, we infuse medium containing SLx at a concentration of $C_{S L x}^{\prime}=$ $10^{-1}$ and either SDS at $C_{\text {SDS }}^{\prime}=5 \times 10^{-2}$, which has no individual activity, or $C_{S D S}^{\prime}=5 \times 10^{-1}$, which has moderate individual activity ( $\square$ and $\square$ with enclosed $\times$, respectively, Figure 4B). Singly, SLx at $C_{\text {SLx }}^{\prime}=10^{-1}$ disrupts $\sim 70 \%$ of the biofilm after $5 \mathrm{~h}$, while both admixtures demonstrate higher potency, removing nearly $100 \%$ of the biofilm by the end of the experiment. For these combinations, disruption occurs both more rapidly and more completely than for either surfactant individually (Figure 7A (upper)). Interestingly, we find that
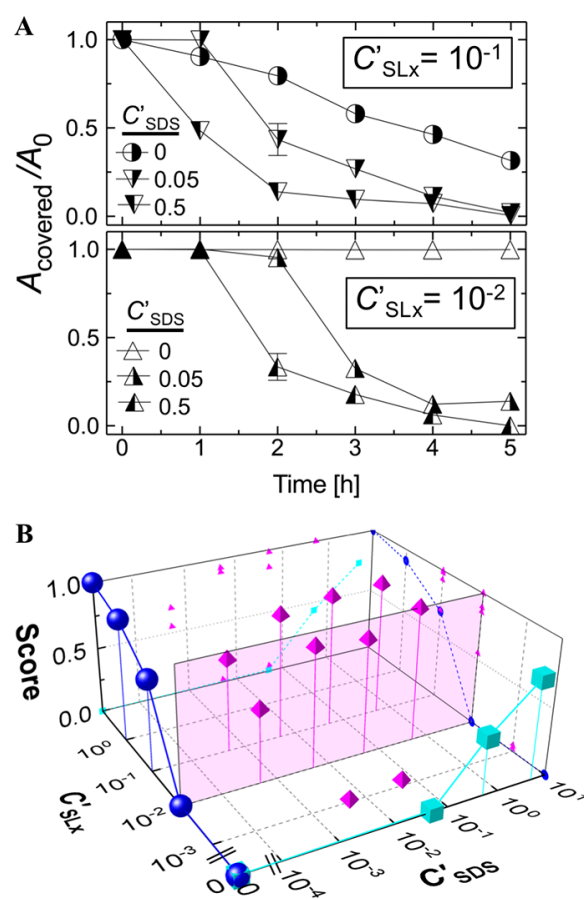

Figure 7. Combined effects of SLx and SDS on biofilm removal from microfluidic channels. (A) The biofilm surface coverage in microchannels as a function of time. In the upper panel, the normalized SLx concentration is $C_{S L x}^{\prime}=10^{-1}$, while the normalized SDS concentrations are $C_{S D C}^{\prime}=0(\mathbf{O}), 0.05(\nabla)$, and $0.5(\boldsymbol{\nabla})$. In the lower panel, $C_{\text {SLx }}^{\prime}=10^{-2}$ and $C_{\text {SDC }}^{\prime}=0(\Delta), 0.05(\boldsymbol{\Delta})$, and $0.5(\Delta)$. In the upper plot, the half-filled circles (O) are data presented in Figure 4B, which are included to aid in comparison. The error bars represent one SD from three experiments, taken using different starting cultures. Error bars smaller than the symbols are omitted for clarity. (B) Biofilm disruption score at $t=5 \mathrm{~h}$ as a function of the normalized concentrations of SLx and SDS. The blue spheres show the effect of SLx alone $\left(C_{S D C}^{\prime}=0\right)$, the cyan cubes show the effect of SDS alone $\left(C_{\text {SLx }}^{\prime}=0\right)$, and the magenta tetrahedra show the effect of the mixtures. The full time-course data for the magenta tetrahedra can be found in Figure 7A and Figure S6. The 2D symbols (circles, squares, and triangles) are projections of the $3 \mathrm{D}$ symbols onto the $\mathrm{XZ}$ and $\mathrm{YZ}$ planes to enable a comparison of the values. The magenta plane indicates the location of the strongest synergistic effects.

decreasing the SLx concentration 10 -fold to $C_{S L x}^{\prime}=10^{-2}$ results in the loss of all biofilm removal activity; however, by supplementing with SDS, the admixture recovers the ability to disrupt $80-100 \%$ of the biofilm depending on the concentration of SDS (Figure 7A (lower)).

To map the combined effects of SLx and SDS, we rate the disruptive capacity of the surfactants at the end of the experiment by defining a score $(0-1)$ given by $1-A_{\text {covered }} / A_{0}$. Here, no disruption has a score of zero, while complete disruption equals unity. We combine the SLx and SDS data from Figures $4 \mathrm{~B}$ and $7 \mathrm{~A}$ with additional microfluidic channel tests (Figure S6). The dose-dependent activity of SLx and SDS, independently, shows that higher concentrations achieve scores near unity whereas lower concentrations approach zero (blue spheres and cyan cubes in Figure 7B). As suggested by the data in Figure 7A, we identify a region where certain SLxSDS admixtures produce significantly higher scores than achieved by their individual components; here, SLx and SDS interact synergistically to boost the score from zero to $\sim 1$ (magenta tetrahedra at $C_{\mathrm{SLx}}^{\prime}=10^{-2}$, Figure $7 \mathrm{~B}$ ). This phase diagram also allows us to determine the lower bound on the 
SLx concentration necessary to achieve biofilm disruption in combination with SDS, which is in the neighborhood of $C_{\mathrm{SLx}}^{\prime}=$ $10^{-3}$.

The demand for biosurfactants with novel functions, biodegradability, and lower toxicity is increasing; ${ }^{25}$ however, the high cost of production ${ }^{20}$ is a major limiting factor. As we demonstrate, admixing high-value biosurfactants with common surfactants may provide a good strategy for improving efficiency as well as potency to meet these demands. It will be important to determine which interactions between different surfactants improve the biofilm disruption activity.

\section{CONCLUSIONS}

In this work, we report on the ability of sophorolipids, a promising biosurfactant, to disrupt $P$. aeruginosa PAO1 biofilms from microfluidic channels. We show that the SLx used here catastrophically disrupts PAO1 WT biofilms at lower concentrations relative to the CMC than observed for SDS, Tween 20, and Tween 80 . We show that despite the strong antibiofilm properties, SLx surprisingly does not appear to have antibacterial effects on PAO1. To shed light on the mechanism of action, we test SLx on an EPS overexpression mutant, finding that SLx causes the detachment of these biofilms from glass surfaces while simultaneously weakening the biofilm structure to the point of disintegration. Although these results demonstrate the potential of SLx, in practice, biosurfactants are costlier to produce than are synthetic surfactants, hindering their use. Thus, there is a need to identify ways to improve potency to decrease production costs. We describe a simple method to boost efficiency: SLx used in combination with SDS not only increases the rate of biofilm disruption relative to SLx or SDS alone but also improves overall microchannel clearance. In combination, both surfactants can be used at concentrations well below the minimum amount required when used individually; this enables a reduction in the dose of surfactants needed and thus the overall cost, and it opens the possibility of safe in vivo administration. Intriguingly, due to this synergy, the concentration of SDS in an SLx-SDS mixture that disrupts $\sim 90 \%$ of a mature PAO1 biofilm is 20 -fold lower than the concentration of SDS found in USFDA-approved interleukin-2 injections, which is $\sim 0.18 \mathrm{mg} / \mathrm{mL} .^{66-68} \mathrm{SLx}-$ SDS mixtures will disrupt $\sim 60 \%$ of a PAO1 biofilm with an SDS concentration that is as low as 200 -fold below $0.18 \mathrm{mg} /$ $\mathrm{mL}$. Identifying cooperative effects between novel biosurfactants and common synthetic surfactants may be a strategy to decrease the environmental impact of the synthetic surfactants while simultaneously retaining the specific functions of both surfactants and possibly opening new avenues to the treatment of bacterial infection. It will be interesting to explore the nature of this synergistic effect in future work.

\section{ASSOCIATED CONTENT}

\section{s) Supporting Information}

The Supporting Information is available free of charge at https://pubs.acs.org/doi/10.1021/acs.langmuir.0c00643.

Detection and identification of the extracted sophorolipids; structures extracted from SLx using LC-MS/ MS analysis; micelle size distributions; conversion between wt \% and values normalized by the CMC; images of disrupted PAO1 biofilms after the start of treatment with a surfactant-laden medium; bactericidal effects of surfactants on PAO1; effect of SLx on PAO1
WT in a shaking culture; and normalized biofilm surface coverage in microchannels as a function of time, under different combinations of SLx and SDS (PDF)

\section{AUTHOR INFORMATION}

\section{Corresponding Authors}

Nobuhiko Nomura - Faculty of Life and Environmental Sciences and Microbiology Research Center for Sustainability (MiCS), University of Tsukuba, Ibaraki 305-8572, Japan; Phone: +81-29-853-6627; Email: nomura.nobuhiko.ge@ u.tsukuba.ac.jp

Andrew S. Utada - Faculty of Life and Environmental Sciences and Microbiology Research Center for Sustainability (MiCS), University of Tsukuba, Ibaraki 305-8572, Japan; 이이. ord 0000-0003-4542-6315; Phone: +81-29-853-4197;

Email: utada.andrew.gm@u.tsukuba.ac.jp

\section{Authors}

Bac V. G. Nguyen - Graduate School of Life and Environmental Sciences, University of Tsukuba, Ibaraki 3058572, Japan; 이이이.org/0000-0003-4510-2660

Toshiki Nagakubo - Faculty of Life and Environmental Sciences, University of Tsukuba, Ibaraki 305-8572, Japan

Masanori Toyofuku - Faculty of Life and Environmental Sciences and Microbiology Research Center for Sustainability (MiCS), University of Tsukuba, Ibaraki 305-8572, Japan

Complete contact information is available at: https://pubs.acs.org/10.1021/acs.langmuir.0c00643

\section{Notes}

The authors declare no competing financial interest.

\section{ACKNOWLEDGMENTS}

This research was generously supported by the Japan Science and Technology Agency (JST), ERATO (JPMJER1502), and a grant-in-aid (17K15410) from the Japanese Society for the Promotion of Science (JSPS). We thank Y. Yamashita and Y. Doan for their help with measuring surface tensions, L. B. Vong for assistance with using the DLS system, M. Prasad for assistance with fabricating microfluidic devices, T. Yamamoto with help with confocal imaging, and J. Fattaccioli for critical feedback.

\section{REFERENCES}

(1) Flemming, H. C.; Wingender, J.; Szewzyk, U.; Steinberg, P.; Rice, S. A.; Kjelleberg, S. Biofilms: An Emergent Form of Bacterial Life. Nat. Rev. Microbiol. 2016, 14, 563-575.

(2) Koo, H.; Allan, R. N.; Howlin, R. P.; Stoodley, P.; Hall-Stoodley, L. Targeting Microbial Biofilms: Current and Prospective Therapeutic Strategies. Nat. Rev. Microbiol. 2017, 15, 740-755.

(3) Eginton, P.; Holah, J.; Allison, D.; Handley, P.; Gilbert, P. Changes in the Strength of Attachment of Micro-Organisms to Surfaces Following Treatment with Disinfectants and Cleansing Agents. Lett. Appl. Microbiol. 1998, 27, 101-105.

(4) Wilking, J. N.; Angelini, T. E.; Seminara, A.; Brenner, M. P.; Weitz, D. A. Biofilms as Complex Fluids. MRS Bull. 2011, 36, 385391.

(5) Romling, U.; Kjelleberg, S.; Normark, S.; Nyman, L.; Uhlin, B. E.; Akerlund, B. Microbial Biofilm Formation: A Need to Act. J. Intern. Med. 2014, 276, 98-110.

(6) Subhadra, B.; Kim, D. H.; Woo, K.; Surendran, S.; Choi, C. H. Control of Biofilm Formation in Healthcare: Recent Advances Exploiting Quorum-Sensing Interference Strategies and Multidrug Efflux Pump Inhibitors. Materials 2018, 11, 1676. 
(7) Al Ashhab, A.; Sweity, A.; Bayramoglu, B.; Herzberg, M.; Gillor, O. Biofouling of Reverse Osmosis Membranes: Effects of Cleaning on Biofilm Microbial Communities, Membrane Performance, and Adherence of Extracellular Polymeric Substances. Biofouling 2017, 33, 397-409.

(8) Whittaker, C.; Ridgway, H.; Olson, B. Evaluation of Cleaning Strategies for Removal of Biofilms from Reverse-Osmosis Membranes. Appl. Environ. Microbiol. 1984, 48, 395-403.

(9) Kip, N.; Van Veen, J. A. The Dual Role of Microbes in Corrosion. ISME J. 2015, 9, 542-551.

(10) Drescher, K.; Shen, Y.; Bassler, B. L.; Stone, H. A. Biofilm Streamers Cause Catastrophic Disruption of Flow with Consequences for Environmental and Medical Systems. Proc. Natl. Acad. Sci. U. S. A. 2013, 110, 4345-4350.

(11) Scheibel, J. J. The Evolution of Anionic Surfactant Technology to Meet the Requirements of the Laundry Detergent Industry. J. Surfactants Deterg. 2004, 7, 319.

(12) Osadebe, A.; Onyiliogwu, C.; Suleiman, B.; Okpokwasili, G. Microbial Degradation of Anionic Surfactants from Laundry Detergents Commonly Discharged into a Riverine Ecosystem. J. Appl. Life Sci. Int. 2018, 16, 1-11.

(13) Marberry, K. M.; Kazmier, P.; Simpson, W. A.; Christensen, G. D.; Phaup, J. G.; Hendricks, K. J.; Anglen, J. O.; Gainor, B. J. Surfactant Wound Irrigation for the Treatment of Staphylococcal Clinical Isolates. Clin. Orthop. Relat. Res. 2002, 403, 73-79.

(14) Van Hamme, J. D.; Singh, A.; Ward, O. P. Physiological Aspects. Part 1 in a Series of Papers Devoted to Surfactants in Microbiology and Biotechnology. Biotechnol. Adv. 2006, 24, 604-620.

(15) Toutain-Kidd, C. M.; Kadivar, S. C.; Bramante, C. T.; Bobin, S. A.; Zegans, M. E. Polysorbate 80 Inhibition of Pseudomonas aeruginosa Biofilm Formation and its Cleavage by the Secreted Lipase LipA. Antimicrob. Agents Chemother. 2009, 53, 136-145.

(16) Das Ghatak, P.; Mathew-Steiner, S. S.; Pandey, P.; Roy, S.; Sen, C. K. A Surfactant Polymer Dressing Potentiates Antimicrobial Efficacy in Biofilm Disruption. Sci. Rep. 2018, 8, 873.

(17) Bondi, C. A.; Marks, J. L.; Wroblewski, L. B.; Raatikainen, H. S.; Lenox, S. R.; Gebhardt, K. E. Human and Environmental Toxicity of Sodium Lauryl Sulfate (SLS): Evidence for Safe Use in Household Cleaning Products. Environ. Health Insights 2015, 9, EHI.S31765.

(18) Cowan-Ellsberry, C.; Belanger, S.; Dorn, P.; Dyer, S.; McAvoy, D.; Sanderson, H.; Versteeg, D.; Ferrer, D.; Stanton, K. Environmental Safety of the Use of Major Surfactant Classes in North America. Crit. Rev. Environ. Sci. Technol. 2014, 44, 1893-1993.

(19) Makkar, R. S.; Rockne, K. J. Comparison of Synthetic Surfactants and Biosurfactants in Enhancing Biodegradation of Polycyclic Aromatic Hydrocarbons. Environ. Toxicol. Chem. 2003, 22, 2280-2292.

(20) Akbari, S.; Abdurahman, N. H.; Yunus, R. M.; Fayaz, F.; Alara, O. R. Biosurfactants-A New Frontier for Social and Environmental Safety: A Mini Review. Biotechnol. Res. Innovation 2018, 2, 81-90.

(21) Marchant, R.; Banat, I. M. Biosurfactants: A Sustainable Replacement for Chemical Surfactants? Biotechnol. Lett. 2012, 34, $1597-1605$.

(22) Marchant, R.; Banat, I. M. Microbial Biosurfactants: Challenges and Opportunities for Future Exploitation. Trends Biotechnol. 2012, $30,558-565$.

(23) Baccile, N.; Babonneau, F.; Banat, I. M.; Ciesielska, K.; Cuvier, A.-S.; Devreese, B.; Everaert, B.; Lydon, H.; Marchant, R.; Mitchell, C. A.; et al. Development of a Cradle-to-Grave Approach for Acetylated Acidic Sophorolipid Biosurfactants. ACS Sustainable Chem. Eng. 2017, 5, 1186-1198.

(24) Diaz De Rienzo, M. A.; Banat, I. M.; Dolman, B.; Winterburn, J.; Martin, P. J. Sophorolipid Biosurfactants: Possible Uses as Antibacterial and Antibiofilm Agent. New Biotechnol. 2015, 32, $720-726$.

(25) Saravanan, V.; Vijayakuma, S. Biosurfactants-Types, Sources and Applications. Res. J. Microbiol. 2015, 10, 181-192.

(26) Rosenberg, E.; Ron, E. High- and Low-Molecular-Mass Microbial Surfactants. Appl. Microbiol. Biotechnol. 1999, 52, 154.
(27) Caiazza, N. C.; Shanks, R. M.; O’Toole, G. A. Rhamnolipids Modulate Swarming Motility Patterns of Pseudomonas aeruginosa. J. Bacteriol. 2005, 187, 7351-7361.

(28) Pamp, S. J.; Tolker-Nielsen, T. Multiple Roles of Biosurfactants in Structural Biofilm Development by Pseudomonas aeruginosa. J. Bacteriol. 2007, 189, 2531-2539.

(29) Joshi-Navare, K.; Prabhune, A. A Biosurfactant-Sophorolipid Acts in Synergy with Antibiotics to Enhance Their Efficiency. BioMed Res. Int. 2013, 2013, 1-8.

(30) Sleiman, J. N.; Kohlhoff, S. A.; Roblin, P. M.; Wallner, S.; Gross, R.; Hammerschlag, M. R.; Zenilman, M. E.; Bluth, M. H. Sophorolipids as Antibacterial Agents. Ann. Clin. Lab. Sci. 2009, 39, $60-63$.

(31) Ribeiro, I. A.; Bronze, M. R.; Castro, M. F.; Ribeiro, M. H. Optimization and Correlation of HPLC-ELSD and HPLC-MS/MS Methods for Identification and Characterization of Sophorolipids. J. Chromatogr. B: Anal. Technol. Biomed. Life Sci. 2012, 899, 72-80.

(32) Penfold, J.; Chen, M.; Thomas, R. K.; Dong, C.; Smyth, T. J.; Perfumo, A.; Marchant, R.; Banat, I. M.; Stevenson, P.; Parry, A.; et al. Solution Self-Assembly of the Sophorolipid Biosurfactant and Its Mixture with Anionic Surfactant Sodium Dodecyl Benzene Sulfonate. Langmuir 2011, 27, 8867-8877.

(33) Pontes, C.; Alves, M.; Santos, C.; Ribeiro, M. H.; Goncalves, L.; Bettencourt, A. F.; Ribeiro, I. A. Can Sophorolipids Prevent Biofilm Formation on Silicone Catheter Tubes? Int. J. Pharm. 2016, 513, 697-708.

(34) Solaiman, D. K. Y.; Ashby, R. D.; Uknalis, J. Characterization of Growth Inhibition of Oral Bacteria by Sophorolipid Using a Microplate-Format Assay. J. Microbiol. Methods 2017, 136, 21-29.

(35) Zhang, X.; Ashby, R. D.; Solaiman, D. K. Y.; Liu, Y.; Fan, X. Antimicrobial Activity and Inactivation Mechanism of Lactonic and Free Acid Sophorolipids Against Escherichia coli O157:H7. Biocatal. Agric. Biotechnol. 2017, 11, 176-182.

(36) Fracchia, L.; Banat, J. J.; Cavallo, M.; Ceresa, C.; Banat, I. M. Potential Therapeutic Applications of Microbial Surface-Active Compounds. AIMS Bioeng. 2015, 2, 144-162.

(37) Diaz De Rienzo, M. A.; Stevenson, P. S.; Marchant, R.; Banat, I. M. Effect of Biosurfactants on Pseudomonas aeruginosa and Staphylococcus aureus Biofilms in a BioFlux Channel. Appl. Microbiol. Biotechnol. 2016, 100, 5773-5779.

(38) Huang, K. C.; Mukhopadhyay, R.; Wen, B.; Gitai, Z.; Wingreen, N. S. Cell Shape and Cell-Wall Organization in Gram-Negative Bacteria. Proc. Natl. Acad. Sci. U. S. A. 2008, 105, 19282-19287.

(39) Kim, S. J.; Chang, J.; Singh, M. Peptidoglycan Architecture of Gram-Positive Bacteria by Solid-State NMR. Biochim. Biophys. Acta, Biomembr. 2015, 1848, 350-362.

(40) Stover, C. K.; Pham, X. Q.; Erwin, A. L.; Mizoguchi, S. D.; Warrener, P.; Hickey, M. J.; Brinkman, F. S. L.; Hufnagle, W. O.; Kowalik, D. J.; Lagrou, M.; et al. Complete Genome Sequence of Pseudomonas aeruginosa PAO1, an Opportunistic Pathogen. Nature 2000, 406, 959-964.

(41) Haagensen, J. A.; Klausen, M.; Ernst, R. K.; Miller, S. I.; Folkesson, A.; Tolker-Nielsen, T.; Molin, S. Differentiation and Distribution of Colistin- and Sodium Dodecyl Sulfate-Tolerant Cells in Pseudomonas aeruginosa Biofilms. J. Bacteriol. 2007, 189, 28-37.

(42) Habash, M. B.; Goodyear, M. C.; Park, A. J.; Surette, M. D.; Vis, E. C.; Harris, R. J.; Khursigara, C. M. Potentiation of Tobramycin by Silver Nanoparticles Against Pseudomonas aeruginosa Biofilms. Antimicrob. Agents Chemother. 2017, 61, e00415.

(43) Huebinger, R. M.; Stones, D. H.; de Souza Santos, M.; Carlson, D. L.; Song, J.; Vaz, D. P.; Keen, E.; Wolf, S. E.; Orth, K.; Krachler, A. M. Targeting Bacterial Adherence Inhibits Multidrug-Resistant Pseudomonas aeruginosa Infection Following Burn Injury. Sci. Rep. 2016, 6, 39341.

(44) Lloyd, M. G.; Lundgren, B. R.; Hall, C. W.; Gagnon, L. B.; Mah, T. F.; Moffat, J. F.; Nomura, C. T. Targeting the Alternative Sigma Factor RpoN to Combat Virulence in Pseudomonas aeruginosa. Sci. Rep. 2017, 7, 12615. 
(45) Zineba, G.; Hassan, L.; Mostafa, M.; Abdellah, H.; Mohammed, T.; El Mostafa, M. Virulence Phenotype, Physicochemical Properties, and Biofilm Formation of Pseudomonas aeruginosa on Polyethylene used in Drinking Water Distribution Systems. Water Resour. 2015, 42, 98-107.

(46) Holloway, B.; Krishnapillai, V.; Morgan, A. Chromosomal Genetics of Pseudomonas. Microbiol. Rev. 1979, 43, 73.

(47) Hickman, J. W.; Tifrea, D. F.; Harwood, C. S. A Chemosensory System that Regulates Biofilm Formation Through Modulation of Cyclic Diguanylate Levels. Proc. Natl. Acad. Sci. U. S. A. 2005, 102, $14422-14427$.

(48) Xia, Y.; Whitesides, G. M. Soft Lithography. Annu. Rev. Mater. Sci. 1998, 28, 153-184.

(49) Mayaud, L.; Carricajo, A.; Zhiri, A.; Aubert, G. Comparison of Bacteriostatic and Bactericidal Activity of 13 Essential Oils Against Strains with Varying Sensitivity to Antibiotics. Lett. Appl. Microbiol. 2008, 47, 167-173.

(50) Singh, A.; Van Hamme, J. D.; Ward, O. P. Surfactants in Microbiology and Biotechnology: Part 2. Application Aspects. Biotechnol. Adv. 2007, 25, 99-121.

(51) Wu, C.; Lim, J. Y.; Fuller, G. G.; Cegelski, L. Disruption of Escherichia coli Amyloid-Integrated Biofilm Formation at the Airliquid Interface by a Polysorbate Surfactant. Langmuir 2013, 29, 920926.

(52) Laatiris, A.; El Achouri, M.; Infante, M. R.; Bensouda, Y. Antibacterial Activity, Structure and CMC Relationships of Alkanediyl Alpha, Omega-Bis(Dimethylammonium Bromide) Surfactants. Microbiol. Res. 2008, 163, 645-650.

(53) Tobe, S.; Majima, T.; Tadenuma, H.; Suekuni, T.; Sakai, K.; Sakai, H.; Abe, M. Nonionic Surfactants Enhancing Bactericidal Activity at their Critical Micelle Concentrations. J. Oleo Sci. 2015, 64, 61-68.

(54) Rosen, M. J.; Li, F.; Morrall, S. W.; Versteeg, D. J. The Relationship Between the Interfacial Properties of Surfactants and their Toxicity to Aquatic Organisms. Environ. Sci. Technol. 2001, 35, 954-959.

(55) Courtney, H. S.; Simpson, W. A.; Beachey, E. H. Relationship of Critical Micelle Concentrations of Bacterial Lipoteichoic Acids to Biological Activities. Infect. Immun. 1986, 51, 414-418.

(56) Kerwin, B. A. Polysorbates 20 and 80 Used in the Formulation of Protein Biotherapeutics: Structure and Degradation Pathways. J. Pharm. Sci. 2008, 97, 2924-2935.

(57) Hirata, Y.; Ryu, M.; Igarashi, K.; Nagatsuka, A.; Furuta, T.; Kanaya, S.; Sugiura, M. Natural Synergism of Acid and Lactone Type Mixed Sophorolipids in Interfacial Activities and Cytotoxicities. J. Oleo Sci. 2009, 58, 565-572.

(58) Kim, H.; Kim, Y.; Lee, B.; Kim, E. Sophorolipid Production by Candida bombicola ATCC 22214 from a Corn-Oil Processing Byproduct. J. Microbiol. Biotechnol. 2005, 15, 55-58.

(59) Zhang, L.; Somasundaran, P.; Singh, S. K.; Felse, A. P.; Gross, R. Synthesis and Interfacial Properties of Sophorolipid Derivatives. Colloids Surf., A 2004, 240, 75-82.

(60) Barken, K. B.; Pamp, S. J.; Yang, L.; Gjermansen, M.; Bertrand, J. J.; Klausen, M.; Givskov, M.; Whitchurch, C. B.; Engel, J. N.; Tolker-Nielsen, T. Roles of Type IV Pili, Flagellum-Mediated Motility and Extracellular DNA in the Formation of Mature Multicellular Structures in Pseudomonas aeruginosa biofilms. Environ. Microbiol. 2008, 10, 2331-2343.

(61) Thullner, M. Comparison of Bioclogging Effects in Saturated Porous Media within One- And Two-Dimensional Flow Systems. Ecol. Eng. 2010, 36, 176-196.

(62) Bereschenko, L. A.; Stams, A. J.; Euverink, G. J.; van Loosdrecht, M. C. Biofilm Formation on Reverse Osmosis Membranes Is Initiated and Dominated by Sphingomonas spp. Appl. Environ. Microbiol. 2010, 76, 2623-2632.

(63) Jerábková, H.; Králová, B.; Náhlák, J. Biofilm of Pseudomonas C12B on Glass Support as Catalytic Agent for Continuous SDS Removal. Int. Biodeterior. Biodegrad. 1999, 44, 233-241.
(64) Ma, L.; Conover, M.; Lu, H.; Parsek, M. R.; Bayles, K.; Wozniak, D. J. Assembly and Development of the Pseudomonas aeruginosa Biofilm Matrix. PLoS Pathog. 2009, 5, No. e1000354.

(65) Rosen, M. J.; Sulthana, S. B. The Interaction of Alkylglycosides with Other Surfactants. J. Colloid Interface Sci. 2001, 239, 528-534.

(66) Crommelin, D. J. A.; Sindelar, R. D.; Meibohm, B. Pharmaceutical Biotechnology, 5th ed.; Springer: 2013.

(67) Lipiainen, T.; Peltoniemi, M.; Sarkhel, S.; Yrjonen, T.; Vuorela, H.; Urtti, A.; Juppo, A. Formulation and stability of cytokine therapeutics. J. Pharm. Sci. 2015, 104, 307-326.

(68) Shin, S.; Ahmed, I.; Hwang, J.; Seo, Y.; Lee, E.; Choi, J.; Moon, S.; Hong, J. W. A Microfluidic Approach to Investigating a Synergistic Effect of Tobramycin and Sodium Dodecyl Sulfate on Pseudomonas aeruginosa Biofilms. Anal. Sci. 2016, 32, 67-73. 